Res., Soc. Dev. 2019; 8(6):e48861082

ISSN 2525-3409 | DOI: http://dx.doi.org/10.33448/rsd-v8i6.1082

\title{
Pesquisa qualitativa em Educação no Brasil: consolidação e desenvolvimento
}

Qualitative research in Education in Brazil: consolidation and development

Investigación cualitativa en Educación en Brasil: consolidación y desarrollo

Recebido: 01/04/2019 | Revisado: 25/04/2019 | Aceito: 08/05/2019 | Publicado: 15/05/2019

Paulo César da Silva Rocha

ORCID: https://orcid.org/0000-0002-1911-7386

Instituto Federal do Ceará (IFCE)

E-mail: paulonihon45@gmail.com

Sandro César Silveira Jucá

ORCID: https://orcid.org/0000-0002-8085-7543

Instituto Federal do Ceará (IFCE), Brasil

E-mail: sandrojuca@ifce.edu.br

Solonildo Almeida da Silva

ORCID: https://orcid.org/0000-0001-5932-1106

Instituto Federal do Ceará (IFCE), Brasil

E-mail: solonildo@ifce.edu.br

Aldayr de Oliveira Monteiro

ORCID: https://orcid.org/0000-0003-3358-5394

Instituto Federal do Ceará (IFCE), Brasil

E-mail: aldayroliveira@alu.ufc.br

\section{Resumo}

Este artigo tem como objetivo discutir como a pesquisa qualitativa desenvolveu-se e consolidou-se no contexto da educação do Brasil, levando em consideração a cronologia dos mais diversos ciclos históricos que deram sustentação aos fundamentos teóricosmetodológicos da pesquisa em Educação no Brasil. A partir deste trabalho empírico bibliográfico que se fundamentou nas diferentes fontes, as quais abordaram reflexões e problematizações das questões metodológicas, demonstrou-se que o pensamento educacional brasileiro passou por diferentes ciclos na história da educação brasileira, tendo configurações que são historicamente comprovadas nas produções dos trabalhos científicos. Para esta discussão, recorreu-se a autores consagrados no que diz respeito a discussão sobre pesquisa qualitativa no contexto educacional Brasileiro, como por exemplo, Marcos Suel Zanette que 
resgata os ciclos históricos da pesquisa qualitativa no Brasil, assim como Bernadete Gatti que traz em suas discussões a relevância dos métodos de pesquisa qualitativa em educação. A temáticas de estudo nas pesquisas qualitativas foram ampliadas ao longo do tempo, assim como a abertura e implantação de mestrados e doutorados, que sofreram influências estrangeiras principalmente no tocante a metodologia. Além disso, houve um grande incentivo que proporcionou esta formação no exterior, tendo como consequência a atuação de professores com especialização em trabalhos acadêmicos, ensino e pesquisa.

Palavras-chave: Pesquisa; Educação; Brasil.

\begin{abstract}
This paper aims to discuss how qualitative research has developed and consolidated in the context of Brazilian education, taking into account the chronology of the most diverse historical cycles that gave support to the theoretical-methodological foundations of the research in Education in Brazil. From this empirical bibliographical work that was based on the different sources, which approached reflections and problematizations of the methodological questions, it was demonstrated that the Brazilian educational thought passed through different cycles in the history of the Brazilian education, having configurations that are historically proven in the productions of the scientific work. For this discussion, consecrated authors were used to discuss qualitative research in the Brazilian educational context, such as Marcos Suel Zanette, who rescues the historical cycles of qualitative research in Brazil, as well as Bernadette Gatti which brings in its discussions the relevance of qualitative research methods in education. The study themes in the qualitative researches were extended over time, as well as the opening and implantation of masters and doctorates, which suffered foreign influences mainly in terms of methodology. In addition, there was a great incentive that provided this training abroad, resulting in the performance of teachers with specialization in academic work, teaching and research.
\end{abstract}

Keywords: Search; Education; Brazil.

\title{
Resumen
}

Este artículo tiene como objetivo discutir cómo la investigación cualitativa se desarrolló y se consolidó en el contexto de la educación de Brasil, teniendo en cuenta la cronología de los más diversos ciclos históricos que dieron sustento a los fundamentos teóricos-metodológicos de la investigación en Educación en Brasil. A partir de este trabajo empírico bibliográfico que se fundamentó en las diferentes fuentes, las cuales abordaron reflexiones y 
problematizaciones de las cuestiones metodológicas, se demostró que el pensamiento educativo brasileño pasó por diferentes ciclos en la historia de la educación brasileña, teniendo configuraciones que son históricamente comprobadas en las producciones de los " trabajos científicos. Para esta discusión, se recurrió a autores consagrados en lo que se refiere a la discusión sobre investigación cualitativa en el contexto educativo brasileño, como por ejemplo, Marcos Suel Zanette, que rescata los ciclos históricos de la investigación cualitativa en Brasil, así como Bernadete Gatti que trae en sus discusiones la relevancia de los métodos de investigación cualitativa en educación. Las temáticas de estudio en las investigaciones cualitativas fueron ampliadas a lo largo del tiempo, así como la apertura e implantación de maestrados y doctorados, que sufrieron influencias extranjeras principalmente en lo tocante a la metodología. Además, hubo un gran incentivo que proporcionó esta formación en el exterior, teniendo como consecuencia la actuación de profesores con especialización en trabajos académicos, enseñanza e investigación.

Palabras clave: Investigación; Educación; Brasil.

\section{Introdução}

O pesquisador, ao longo da vida acadêmica, tem a necessidade de definir caminho que irá trilhar durante sua pesquisa. É necessária uma discussão sobre o percurso metodológico que deverá ser percorrido a fim de haver a transformação do fenômeno investigado em objeto de pesquisa. Este artigo tem como objetivo discutir como a pesquisa qualitativa desenvolveuse e consolidou-se no contexto da educação do Brasil, levando em consideração a cronologia dos mais diversos ciclos históricos que deram sustentação aos fundamentos teóricosmetodológicos da pesquisa em Educação no Brasil.

Este artigo tem como objetivo discutir como a pesquisa qualitativa desenvolveu-se e consolidou-se no contexto da educação do Brasil, levando em consideração a cronologia dos mais diversos ciclos históricos que deram sustentação aos fundamentos teóricosmetodológicos da pesquisa em Educação no Brasil. A partir deste trabalho empírico bibliográfico que se fundamentou nas diferentes fontes, as quais abordaram reflexões e problematizações das questões metodológicas, demonstrou-se que o pensamento educacional brasileiro passou por diferentes ciclos na história da educação brasileira, tendo configurações que são historicamente comprovadas nas produções dos trabalhos científicos.

De acordo com Zanette (2017)), no Brasil, as abordagens das pesquisas qualitativas configuram-se, como enfoque metodológico, a partir da década de 1970, devido às 
Res., Soc. Dev. 2019; 8(6):e48861082

ISSN 2525-3409 | DOI: http://dx.doi.org/10.33448/rsd-v8i6.1082

concepções epistemológicas interpretarem a realidade de forma distorcida nas suas metodologias. O autor pontua que neste caminho percorrido ao longo da história, percebeu-se uma maior preocupação com o método que seria empregado do que com o próprio questionamento a ser elucidado no contexto da Educação.

As distorções quantitativas são devidas à precariedade das fontes, à manipulação da informação social, à imprecisão das técnicas em excluir certas variáveis para a explicitação do fenômeno escola, por exemplo. Na concepção positivista tradicional, a objetividade é desejada, por meio da quantificação, como modo de eliminar as distorções devidas à subjetividade do pesquisador. Nesse período, esse modelo de ciência passa a receber severas críticas filosóficas, políticas e técnicas. O alvo dessas críticas direcionou-se para a aplicabilidade dos modelos de ciências naturais nas Ciências Sociais e Humanas, cujos princípios teóricos separavam os fatos dos seus contextos histórico-culturais. (Zanette, 2017, p. 154)

Ressalta-se, primeiramente, que dois aspectos proporcionaram o desenvolvimento da pesquisa em Educação no Brasil. Um destes fatores está relacionado as pesquisas que tiveram início nas Ciências Sociais e Humanas em vários países, influenciando grupos de pesquisas no Brasil, que até então apontavam uma defasagem neste tipo de pesquisa. Outro aspecto importante que pode ser mencionado foi a expansão das pós-graduações no Brasil, que tiveram como objetivo focar na qualificação dos profissionais de Educação, justificando este investimento que teve como apoio fontes financiadoras.

\section{Metodologia}

Para esse artigo foi realizado uma pesquisa qualitativa, que, de acordo com Minayo (2007) possibilita ao pesquisador uma análise aprofundada do objeto estudado. Inicialmente, foi utilizada a pesquisa exploratória para resgatar os ciclos históricos pelos quais a pesquisa em Educação perpassou, assim como as implicações e influências sofridas ao longo do tempo.

A segunda etapa desta pesquisa foi o levantamento bibliográfico, delimitando aos fatos que estavam relacionados diretamente com pesquisa em Educação, principalmente no tocante as pesquisas qualitativas. Um levantamento bibliográfico é um trabalho conceitual, investigativo, extremamente minucioso e eixo indispensável para toda construção da pesquisa. 
ISSN 2525-3409 | DOI: http://dx.doi.org/10.33448/rsd-v8i6.1082

A análise de conteúdo foi dividia em três etapas: pré-análise dos textos, com a leitura do material bibliográfico; exploração do material, e tratamento dos resultados obtidos, com a reflexão e a interpretação sobre os dados, produzindo o quadro de dados dos ciclos históricos da pesquisa em Educação no Brasil.

\begin{tabular}{|c|c|}
\hline Período & Ciclos da pesquisa qualitativa em Educação \\
\hline Antes de 1938 & $\begin{array}{l}\text { Estudos em Educação expressavam-se apenas pelo trabalho isolado de } \\
\text { alguns professores da área. }\end{array}$ \\
\hline 1938 & $\begin{array}{l}\text { Produção de modo mais regular no Brasil com a implantação do INEP, } \\
\text { que se desdobrou em Centro Brasileiro de Pesquisas Educacionais } \\
\text { (CBPE) e nos Centros Regionais }\end{array}$ \\
\hline 1940 & publicação da Revista Brasileira de Estudos Pedagógicos (RBPE-INEP) \\
\hline 1950 & $\begin{array}{l}\text { Publicação das séries "Monografias", "Estudos", "Documentos", } \\
\text { "Pesquisas e Planejamento", Atualidades Pedagógicas da Editora } \\
\text { Civilização Brasileira (1950), a Revista Pedagógica, }\end{array}$ \\
\hline 1955 & Publicação da Revista Pedagógica, da Universidade de São Paulo \\
\hline 1960 & $\begin{array}{l}\text { Expansão do quadro das universidades. Alguns grupos de pesquisa } \\
\text { emergiram devido a implantação de cursos de pós-graduação. }\end{array}$ \\
\hline 1968 & Primeiros programas de pós-graduação em Educação (strictu sensu) \\
\hline 1971 & Criação de dez cursos de pós-graduação em Educação (strictu sensu) \\
\hline 1975 & $\begin{array}{l}\text { Já havia dezesseis cursos de pós-graduação em Educação (strictu sensu) } \\
\text { instalados }\end{array}$ \\
\hline 1970-1976 & $\begin{array}{l}\text { as pesquisas em Educação figuram sobre os "relatos de experiências ou } \\
\text { tentativa de renovação educacional" com "estudos descritivos" }\end{array}$ \\
\hline 1980 & $\begin{array}{l}\text { aparecem trabalhos sobre "política educacional, tema até então ausente } \\
\text { em trabalhos de análise institucional e organizacional" }\end{array}$ \\
\hline
\end{tabular}




\begin{tabular}{|l|l|}
\hline 1981 & $\begin{array}{l}\text { foram a implantados } 27 \text { programas de pós-graduação em Educação, } \\
\text { funcionando em } 27 \text { instituições de ensino superior, que resultaram na } \\
\text { produção de centenas de dissertações e de dez teses de doutorado, } \\
\text { concluídas até } 1981 .\end{array}$ \\
\hline
\end{tabular}

É importante ressaltar que esta tabela não exclui outros dados encontrados por outros pesquisadores no período anterior a 1930, e que não foram contemplados na obra de Zanette (2017) ou neste artigo.

Outro dado complementar diz respeito a não continuidade da sequência das revistas publicadas entre 1940 e 1960, em que não tiveram continuidade. Apesar disto, estas publicações tiveram sua contribuição com a pesquisa na área de Educação, divulgando e aplicando práticas pedagógicas, assim como teorias metodológicas. Outro ponto a se destacar é a criação dos mestrados em Educação por meio dos programas de pós-graduação, que até 1975 já haviam dezesseis em funcionamento.

\section{Resultados e discussões}

Segundo Zanette (2017), quando o Instituto Nacional de Estudos Pedagógicos (INEP) foi criado, em 1938, teve papel importante na produção de métodos e técnicas de investigação científica em Educação por meio dos Centro Brasileiro de Pesquisas Educacionais (CBPE) e nos Centros Regionais em alguns estados, como Rio Grande do Sul, São Paulo, Bahia e Minas Gerais. Para o autor, estes órgãos tiveram papel importante ao proporcionar um espaço específico de produção e de estimular de forma sistemática a pesquisa em Educação. Gatti (1983) ressalta que até então, os trabalhos em Educação limitavam-se a trabalhos isolados de alguns professores da área.

Ao mesmo tempo, recursos humanos formados em Educação eram especializados por meio das Escolas Normais de alto nível. Despontando posteriormente em cursos de pedagogia, e anos mais tarde com pós-graduação em Educação.

O sistema de pós-graduação brasileiro, iniciado em 1965 a partir do parecer n. 977/65 do Conselho Federal de Educação, vivenciou rápida expansão como parte de um projeto de Estado que considerava o desenvolvimento científico "pré-condição para o desenvolvimento econômico" (Brasil, 1983, p. 7). De acordo com Córdova, Gusso e Luna (1986), esta expansão rápida e desorganizada do sistema de pós-graduação fez com que cada curso 
valorizasse uma das funções que se entendia ter a pós-graduação: a formação de professores para o ensino superior, a capacitação de técnicos para operar os projetos governamentais e a produção de conhecimentos tendo em vista ao desenvolvimento econômico do país.

Ao mesmo tempo em que os cursos tinham perfis diferenciados devido a essas funções, os currículos apresentavam homogeneidade. Os cursos organizavam-se em torno de áreas de concentração e de um conjunto de disciplinas obrigatórias de domínio conexo e eletivas, sem grande valorização da pesquisa, tanto docente quanto como atividade orgânica dos programas. Para Fávero (1996), as recomendações dos pareceres n. 977/65 e n. 77/69, embora não obrigatórias, foram seguidas à risca pelos cursos, na medida em que era a partir delas que seu funcionamento era autorizado, condição fundamental para a validação dos certificados e para pleitear financiamento junto aos órgãos estatais.

Um aspecto importante também justifica a intensidade de publicações, assim como a qualidade referente a elas: a qualificação acadêmica em programas de pós-graduação fora do Brasil, tanto programa de mestrado, como de doutorado. Houve um grande incentivo que proporcionou esta formação no exterior, tendo como consequência a atuação de professores com especialização em trabalhos acadêmicos, ensino e pesquisa. Gatti (1983) aponta que os temas foram ampliados e diversificados, assim como diversas mudanças, tanto nos temas, como nas abordagens metodológicas. Em decorrência destas mudanças, ampliações e diversificações, existiram ciclos., convergências acerca das temáticas e das metodologias.

Gouveia (1976) salienta que antes as pesquisas concentravam-se mais em abordagens de teor psico-pedagógico, abordando processo de ensino, aprendizagem, desenvolvimento metodológico, etc. Entretanto, a partir da década de cinquenta, o foco passa a ser o desenvolvimento da sociedade brasileira, condições culturais, ou seja, o foco passa a figurar em aspectos da relação entre o sistema escolar e determinados aspectos sociais. Ainda segundo a autora, na década de sessenta, são publicados trabalhos acerca de educação como formação de recursos humanos, num viés de natureza econômica, de demanda profissional.

Segundo Gatti (1983), em 1970, as temáticas de estudo foram ampliadas, com enfoque em currículos, avaliação de programas, caracterizações de redes e recursos educativos, relações de educação e trabalho, características de alunos, famílias e ambiente de que provêm, nutrição e aprendizagem, validação e critica de instrumentos de diagnósticos e a validação, estratégia de ensino entre outros. Gouveia (1976) complementa que as pesquisas em educação 
no período de 1970 a 1976 versaram mais em estudos descritivos acerca da tentativa de renovação educacional ou mesmo relatos de experiência.

Gonçalves (1984) aponta que as publicações na área de Educação indicavam grande inquietação, em que o foco do debate na academia estava relacionado na escolha de determinada metodologia em detrimento de outra. Parte dos pesquisadores defendiam determinadas técnicas para coletar os dados da pesquisa, assim como analisar os dados. De acordo com o autor, neste momento específico, a busca por métodos intercomplementares era crescente, sendo bem vista a relação entre pesquisa quantitativa e qualitativa.

Um tema até então inexistente, sobre política educacional, apareceu em trabalhos na década de 80, e um dos fatores que possibilitaram esta produção foi o fato de 27 programas de pós-graduação em Educação terem sido implantados, em 27 instituições de ensino superior "que resultaram na produção de centenas de dissertações e de dez teses de doutorado, concluídas até 1981.” (Zanette, 2017, p. 153).

Macedo e Sousa (2010) entendem que a pós-graduação e a pesquisa na área de educação, ao longo dos últimos trinta anos, viveu intensa consolidação, em que se percebe, entre outros fatores, pelas demandas que foram ampliadas por financiamento em diversas agências, tratando-se de uma trajetória comum a outras áreas das ciências sociais e das humanidades. Velho (1997) ressalta por vezes faz parecer desestruturação, o que é uma "saudável vitalidade da comunidade" científica na competição por recursos que não foram ampliados na mesma medida em que aumentaram as demandas.

Conforme esse autor (idem, p. 5), a maior institucionalização do sistema provocou algum desconforto pela maior competitividade por recursos, mas foi "absolutamente crucial para que a pesquisa propriamente dita desse um salto" e as áreas consolidassem-se

Do ponto de vista da ampliação do sistema de pós-graduação, parece que estamos atingindo certa saturação. A capacidade de criação de novos cursos de mestrado vem-se reduzindo e concentrando-se em universidades privadas. No nível de doutorado, ainda há algum fôlego, mas em geral os cursos novos contam com reduzido número de docentes, o que deve dificultar-lhes atingir níveis de excelência. Parece estarmos num momento em que é fundamental projetar estratégias para a continuidade da ampliação quantitativa e qualitativa da área. Pensar em associações é talvez uma forma de garantir o crescimento do sistema sem 
Res., Soc. Dev. 2019; 8(6):e48861082

ISSN 2525-3409 | DOI: http://dx.doi.org/10.33448/rsd-v8i6.1082

perda da qualidade, facilitando a consolidação de novos programas que venham a atender a enorme demanda por formação pós-graduada stricto sensu na área da educação (Macedo \& Sousa, 2010, p. 169).

Desta forma, entende-se que passa-se a existir uma concentração nas universidades privadas no tocante a cursos de mestrados, possivelmente indicando um crescimento substancial, mas sem qualidade. Esta demanda certamente acontece devido a grande demanda por formação de pós-graduação exigida pelo mercado de trabalho.

\section{Considerações Finais}

A partir deste trabalho empírico bibliográfico que se fundamentou nas diferentes fontes, as quais abordaram reflexões e problematizações das questões metodológicas, demonstrou-se que o pensamento educacional brasileiro passou por diferentes ciclos na história da educação brasileira, tendo configurações são historicamente comprovadas nas produções dos trabalhos científicos. Além disso, concluímos que dois aspectos proporcionaram o desenvolvimento da pesquisa em Educação no Brasil. Um destes fatores está relacionado as pesquisas que tiveram início nas Ciências Sociais e Humanas em vários países, influenciando grupos de pesquisas no Brasil, que até então apontavam uma defasagem neste tipo de pesquisa. Outro aspecto importante que pode ser mencionado foi a expansão das pós-graduações no Brasil, que tiveram como objetivo focar na qualificação dos profissionais de Educação

A temáticas de estudo foram ampliadas ao longo do tempo, assim como a abertura e implantação de mestrados e doutorados, que sofreram influências estrangeiras principalmente no tocante a metodologia. Além disso, houve um grande incentivo que proporcionou esta formação no exterior, tendo como consequência a atuação de professores com especialização em trabalhos acadêmicos, ensino e pesquisa.

Sugere-se para trabalhos um aprofundamento no que diz respeito a pesquisa educacional entre 2010 e 2019, elaborando um paralelo entre o desenvolvimento da pesquisa educacional no Brasil nos ciclos apresentados neste artigo, dando subsídios para a leitura e análise de um panorama atualizado de forma a sustentar ações para consolidar a pesquisa qualitativa na educação brasileira. 


\section{Referências}

Brasil. Desenvolvimento científico e formação de recursos humanos. Brasília: CNPq, 1983.

Córdova, R., Gusso, D., Luna, S. pós-graduação na América Latina: o caso brasileiro. Brasília: UNESCO/CRESALC/MEC/ SESu/CAPES, 1986.

Fávero, O. Situação atual e tendências de reestruturação dos programas de pós-graduação em educação. Revista da Faculdade de Educação da USP, v. 22, n. 1, p. 51-88, jan./jun. 1996.

Gatti, B. A. Pós-graduação e pesquisa em educação no Brasil, 1978-1981. Cadernos de Pesquisa, n. 44, p. 03-17, 1983.

Gatti, B. A.; ANDRÉ, M. A relevância dos métodos de pesquisa qualitativa em educação no Brasil. In: WELLER, W.; PFAFF, N. (Orgs.). Metodologias da pesquisa qualitativa em Educação: teoria e prática. 2. ed. Petrópolis: Vozes, 2011. p. 29-38.

Gonçalves, O. Incorporação de práticas curriculares nas escolas. CP Cadernos de Pesquisa, FCC, n. 49, p. 55-62, 1984.

Gouveia, A. J. A pesquisa sobre educação no Brasil: de 1970 para cá. Cadernos de Pesquisa, n. 19 , p. $75-9,1976$.

Macedo, E; Sousa, C. A pesquisa em educação no Brasil. Revista Brasileira de Educação, Rio de Janeiro, v. 15, n. 43, p.166-202, jan. 2010.

Minayo, M. C. S. O desafio do conhecimento. Pesquisa qualitativa em saúde. São Paulo: HUCITEC, 2007. Autores, que tal utilizar autores mais recentes e voltados para educação, por exemplo vejam as sugestões seguintes e que falam a mesma coisa em relação às pesquisas qualitativas:

Velho, G. As ciências sociais nos últimos 20 anos: três perspectivas. Revista Brasileira de Ciências Sociais, v. 12, n. 35, p. 1-18, fev. 1997.

ZANETTE, Marcos Suel. Pesquisa qualitativa no contexto da Educação no Brasil. Educar em Revista, n. 65, p. 149-166, 2017. Disponível em: . 
Res., Soc. Dev. 2019; 8(6):e48861082

ISSN 2525-3409 | DOI: http://dx.doi.org/10.33448/rsd-v8i6.1082

Porcentagem de contribuição de cada autor no manuscrito

Paulo César da Silva Rocha $-40 \%$

Sandro César Silveira Jucá - 30\%

Solonildo Almeida da Silva - 20\%

Aldayr de Oliveira Monteiro - 10\% 